\title{
Floristics and Reproductive Phenology of Trees and Bushes in Central West Brazil
}

\author{
VIVIAN A. ASSUNÇÃO ${ }^{1}$, JOSÉ C. CASAGRANDE ${ }^{2}$ and ÂNGELA L.B. SARTORI ${ }^{1}$ \\ ${ }^{1}$ Universidade Federal de Mato Grosso do Sul, Centro de Ciências Biológicas e da Saúde, \\ Programa de Pós-Graduação em Biologia Vegetal, Laboratório de Botânica, Caixa Postal 549, 79070-900 Campo Grande, MS, Brasil \\ ${ }^{2}$ Universidade Federal de São Carlos, Centro de Ciências Agrárias, \\ Departamento de Recursos Naturais e Proteção Ambiental, Caixa Postal 153, 13600-970 Araras, São Paulo, Brasil
}

Manuscript received on February 6, 2013; accepted for publication on September 11, 2013

\begin{abstract}
Environmental conditions such as temperature, soil, photoperiodic factors and precipitation can determine the physical environment favoring the occurrence of given species and interfere with the reproductive period of plants. This work involved monthly excursions to a slope in the Serra de Maracaju between December 2009 and November 2010. Samples were collected in eight $25 \times 50 \mathrm{~m}$ plots, transverse to the slope, totaling 1 ha sampled. Floristic richness included 79 species distributed into 58 genera and 31 families. Floristic Similarity Analysis and Principal Component Analysis showed the occurrence of two plant formations on the slope: seasonal deciduous forest and cerradão on the eutrophic soil of the slope. Flowering and fruiting occured in the rainy season; correlation with rainfall, mean temperature and photoperiod were negative for flowering and positive for fructification. Trees and bushes presented uniform flowering and a moderately seasonal fructification, which is unexpected in seasonal environments. We discuss the implications of the patterns found and make comparisons with others studies.
\end{abstract}

Key words: Cerradão, community, eciduous forest, eutrophic soil.

\section{INTRODUCTION}

Environmental conditions such as temperature, soil, photoperiodic factors and precipitation can determine the physical environment favoring the occurrence of given species and interfere with the reproductive period of plants (Towsend et al. 2006). In tropical and subtropical regions, phenological events are mainly associated with rain (Frankie et al. 1974) and soil moisture conditions (Morellato et al. 2000). In temperate areas, phenophases depend more on length and temperature of the day (Opler et al. 1976).

Correspondence to: Vivian Almeida Assunção

E-mail: vivian.bios@gmail.com
The Cerrado, a Savanna-like formation, is the second most important Brazilian morphoclimatic zone (Rodrigues 2005), gathering different natural phytophysiognomies (Eiten 1982), according to local edaphic, topographic and climatic factors (Oliveira and Marquis 2002). Its significant species richness may be surrounded by different vegetation types such as Amazonian and Atlantic forests, thorn forest (Chaco) and caatinga, subtropical fields, and wet and dry areas (Simon et al. 2009).

The fact that dry forests are interspersed with Cerrado in great tracts of land throughout South America (Mendonça et al. 1998) can be explained 
by climatological events that go back to the typically dry Quaternary period, which would have enabled the expansion of these forests and of more opened plant formations as fields, Cerrado and caatinga. Areas with dry forests differ in that they have more fertile soils, a higher $\mathrm{pH}$ and a concentration of certain nutrients (Mendonça et al. 1998).

Cerrado and forest remnants are often interspersed on Brazilian Serra slopes in the states of Minas Gerais (Oliveira-Filho et al. 1994), Goiás (Felfili et al. 2007) and Mato Grosso do Sul (Salis et al. 2004, G.A. Damasceno-Júnior, unpublished data, B.G. Fina, unpublished data, Ramos and Sartori 2013). More recent rugged landscapes are usually richer in given soil elements, which can favor the settlement of different, albeit geographically close plants (Reatto et al. 2008). Phenological studies at a community level can help functionally classify vegetation (Vasconcelos et al. 2010) and are crucial to understand plant reproduction and ecology (Oliveira 2008).

Studies in Venezuelan savanna and semideciduous forest communities report that most species blossom in the rainy season and in the rainy and dry seasons, respectively (Monasterio and Sarmiento 1976). In Cerrado communities located in different Brazilian regions, flowering usually occurs at the beginning of the rainy season (Batalha and Mantovani 2000) or, more rarely, during the dry season (Miranda 1995). However, other studies suggest that in transition periods flowering intensity in the Cerrado could be influenced by photoperiod (Batalha and Martins 2004) or by the association of the biological features of Cerrado species (Sarmiento and Monasterio 1983) and edaphic conditions (Vasconcelos et al. 2010).

Fruit types and, consequently, dispersal modes also seem to be associated with climatic factors (Morellato and Leitão-Filho 1992, Batalha and Martins 2004). In Cerrado communities, zoochory prevails during the rainy season, while auto- and anemochory are more frequent in the dry period (Morellato and Leitão-Filho 1992, Batalha and Martins 2004, Miranda 1995).

The basalt plateau of the Paraná Basin extends from South-Central Brazil to Uruguay. Within the state of Mato Grosso do Sul in West-Central Brazil, it comprises a set of escarpments called the Serra de Maracaju (Martins 2003). Although the southern part of this Serra has rugged landscapes and steep hillsides with different altitudes, phytophysiognomies, and floristic compositions, it has been scarcely studied (B.G. Fina, unpublished data, Ramos and Sartori 2013) and no work has conjointly addressed its floristic and phenological aspects.

Based on the physical proximity of the vegetation growing on the same side of this Serra de Maracaju slope and considering that they are part of the Cerrado area, we hoped to find both different formations influenced by soil fertility and reproductive phenophases regulated by abiotic factors such as rainfall, day length and temperature (monthly means).

The purpose of this study was to discover 1) if the floristic composition corroborates the fact that different phytophysiognomies grow on the slope, 2) whether there is a relation between soil fertility and floristics, 3) if flowering, fructification and dispersal syndromes are uniform throughout the year, and 4) whether flowering and fructification periods relate to abiotic factors.

\section{MATERIALS AND METHODS}

General CharacterizATION OF THE StUdy AREA

Study area is located $47 \mathrm{~km}$ from Campo Grande, Mato Grosso do Sul, Brazil, between $20^{\circ} 05^{\prime}$ to $20^{\circ} 30^{\prime} \mathrm{S}$ and $54^{\circ} 45^{\prime}$ to $54^{\circ} 15^{\prime} \mathrm{W}$, at an approximate altitude of $500 \mathrm{~m}$ above sea level (see Figure 1). Vegetation is essentially Cerrado interspersed with seasonal forests (RADAMBRASIL 1982), which, according to UNESCO (1973), are classified as tropical and subtropical drought-deciduous forests. The study area is located in the Serra de Maracaju, which practically crosses the state longitudinally 
and is characterized by significant topographic variations, a great quantity of fountainheads, springs and two main watercourses: the Paraguay and the Paraná Rivers.

Local soils are classified as dystrophic purple latosol and dark red alic and litholic eutrophic latosol (Oliveira et al. 2006). The slope studied here is not deforested and evidence shows it has not burnt for at least 50 years, but cattle grazes in some of its parts. Climate is hot and dry, classified as Aw (Köppen 1948), with rain throughout the year and a mean rainfall.

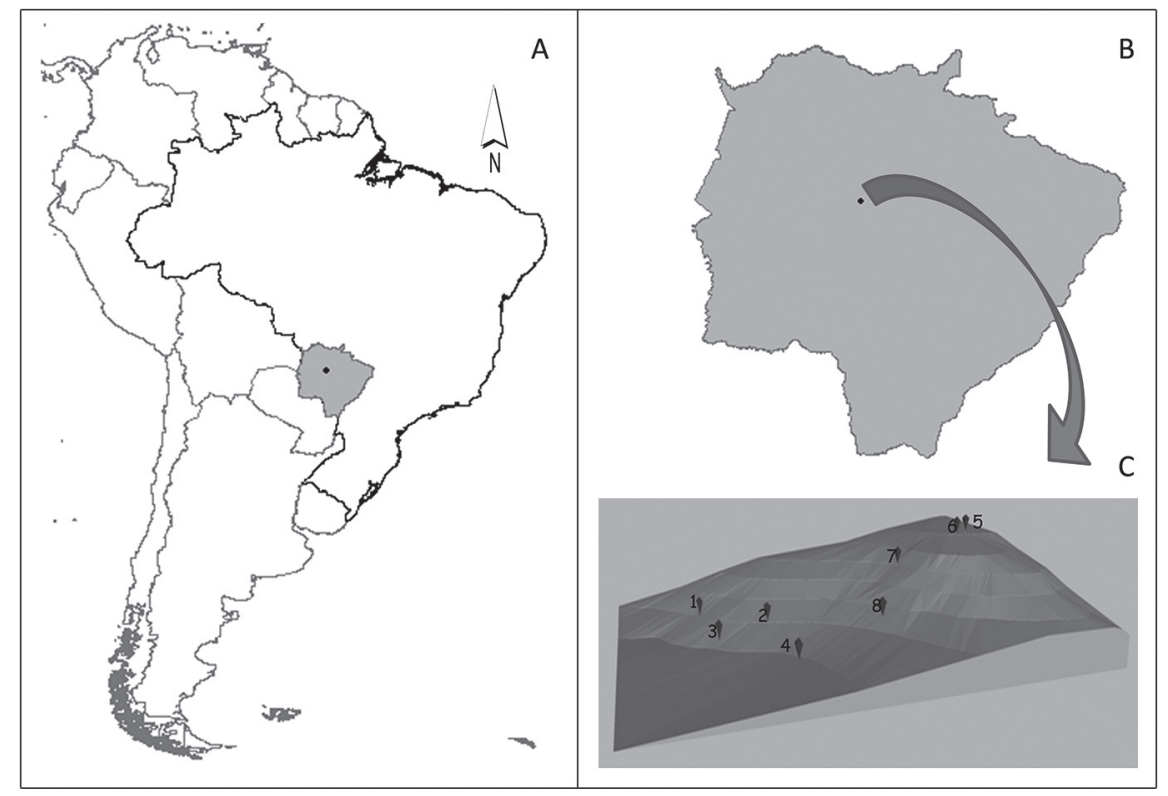

Figure 1 - Slope studied in the Serra de Maracaju (C), Jaraguari (black point) (B), South America (white), Mato Grosso do Sul, Brazil (gray) (A). (in Figure C, 1,2,3,4,5,6,7,8 identify the plots).

\section{DATA COLLECTION AND ANALYSIS}

In 34 ha covered with native vegetation, eight $25 \times 50 \mathrm{~m}$ plots transverse to slope were delimited (see Figure 1), totaling 1 ha sampled. All trees and bushes were sampled in each plot. To complete the floristic survey, fertile materials were collected during random walks near each plot.

Flowering and fructification periods of bush and tree individuals were followed up in those plots at community level and recorded monthly on a spreadsheet (presence/absence) between December 2009 and November 2010; the maturity stages of flowers (bud and pre-anthesis) and fruits were disregarded.

Species were assorted by habit according to Cain and Castro (1959), modified by Whittaker
(1975): trees are woody plants over $3 \mathrm{~m}$ high and bushes are 1-3 m high. At family level, nomenclature follows APG III (2009). Materials were deposited at the CGMS herbarium. Dispersal syndromes classification, into anemochoric (winged diaspores), zoochoric (by animals) and autochoric (by explosion or gravity), follows Pijl (1982).

To compare the floristic data obtained in our plots, we used the Sørensen similarity index (MüllerDombois and Ellenmberg 1974). A dendrogram was constructed based on the group mean values (UPGMA). Spearman correlation analysis (Zar 1984) was used to ascertain the relations between monthly rainfall, day lenght and temperature (monthly means) and flowering and fructification, during the year of study and between 2000 and 2010. The relation of 
the day length with flowering and fructification, was estimated only for the year study. The abiotic data used in the correlation analyses of the year of study were those measured 2 months before collection, since the response of plants to the effect of climate is usually delayed (Marques et al. 2004)

We also checked if flowering and fruiting were different between floristic groups, through the MannWhitney test (Zar 1984), if the proportions of anemo-, zoo- and autochory were different by performing a chi-square test (Zar 1984), and, using species number, if flowering, fructification and dispersal syndromes were uniform throughout the year, using circular statistics (Zar 1984); circular statistics is highly recommended to investigate data generated from circular scales (Morellato et al. 2010).

Monthly mean day lengths were obtained from the Brazilian Observatory (Moreira 2011). A climatogram was created according to Walter (1986). Monthly mean rainfall and temperature from 2000 to 2010 was provided by the Center for Meteorological Analyses of the Universidade Anhanguera-UNIDERP (University for the Development of the State and of the Pantanal Region), which is located $32 \mathrm{~km}$ away from our study site $\left(20^{\circ} 26^{\prime} 19.4\right.$ ' $\mathrm{S}$; 5432'18.3" W; $669 \mathrm{~m}$ ); monthly rainfall below $100 \mathrm{~mm}$ was considered the least wet season. The cumulative rainfall of the year of study was $1,673.5 \mathrm{~mm}$, which is higher than the $1,468.8 \mathrm{~mm}$ mean of the previous 10 years. Mean temperature of the year was $23.6^{\circ} \mathrm{C}$ and that of the previous 10 years was $23.4^{\circ} \mathrm{C}$.

Our climatogram (see Figure 2) showed that the wet season runs from September to March, whereas the period from April to August is less humid, although there is no water deficit. The monthly mean average rainfall during the 10 previous years was $122.4 \mathrm{~mm}$, while that of the year of study was $139.5 \mathrm{~mm}$.

Fifteen soil samples were collected in each plot, 0-20 cm deep. Sampled points were randomly

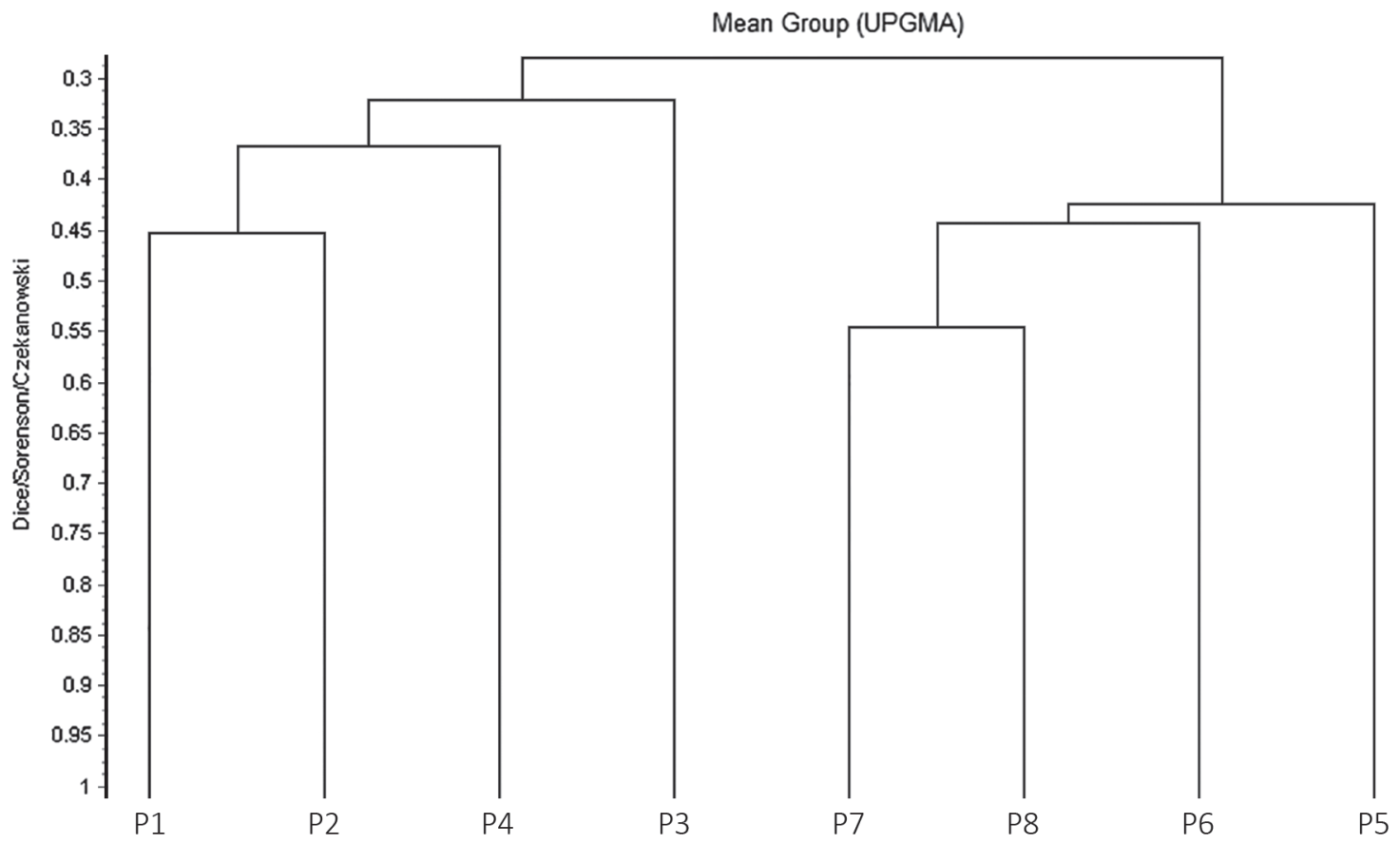

Figure 2 - Climatic diagram (2000 to 2010) built from the data obtained from the Center for Meteorological Analyses of the University for the Development of the State and of Pantanal Region (Universidade Anhanguera-UNIDERP), Mato Grosso do Sul, Brazil. 
chosen along each plot to constitute a one and only sample. Contents of macro- and micronutrients, and Al, Cation Exchange Capacity (CEC), pH, organic matter, $\mathrm{V} \%$ and $\mathrm{BS}$ (base sum) were assessed. The analyses were performed at the laboratory of the Federal University of São Carlos at Araras, SP, Brazil. When comparing soil features, we performed a Principal Component Analysis (PCA) with 13 variables, excluding the graphic representations of $\mathrm{H}+\mathrm{Al}, \mathrm{BS}, \mathrm{CEC}$ and $\mathrm{V} \%$.

\section{RESULTS}

Floristic richness included 75 species distributed into 58 genera and 31 families (see Table I). In the plots, 54 species belonging to 40 genera and 23 families were sampled (see Table II). The families with highest species richness were: Fabaceae, $10 \mathrm{spp}$ or $13.3 \%$, Myrtaceae and Malvaceae, seven spp or $9.3 \%$ each (see Table I). The remaining families were represented by 51 species (see Table I).

TABLE I

List of families and species present in the plots and respective growth habits on an escarpment slope of the Serra de Maracaju, Mato Grosso do Sul, Brazil. (* species obtained in the plots, ** vegetative material; plots: P1, P2, P3, P4, P5, P6, P7 and P8; Voucher - herbarium CGMS).

\begin{tabular}{|c|c|c|c|c|c|c|c|c|c|c|c|}
\hline Family & Species & $\begin{array}{c}\text { Growth } \\
\text { habit }\end{array}$ & $\mathbf{P 1}$ & $\mathbf{P 2}$ & P3 & $\mathbf{P 4}$ & P5 & P6 & P7 & P8 & Voucher \\
\hline \multirow{3}{*}{ Anacardiaceae } & $\begin{array}{l}\text { Astronium fraxinifolium Schott ex } \\
\text { Spreng. }\end{array}$ & Tree & $\mathrm{X}$ & & & & $\mathrm{X}$ & $\mathrm{X}$ & & & 31398 \\
\hline & Lithraea molleoides Engl. & Tree & & & $\mathrm{X}$ & & $\mathrm{X}$ & & $\mathrm{X}$ & $\mathrm{X}$ & 31399 \\
\hline & Myracrodruon urundeuva Allemão* & Tree & & & & & & & & $\mathrm{X}$ & 31400 \\
\hline \multirow{3}{*}{ Annonaceae } & Annona cornifolia A. St.-Hil. & Shrub & & & & & & & & & 31401 \\
\hline & Annona crassiflora Mart. & Shrub & & & & & $\mathrm{X}$ & & & & 31402 \\
\hline & Rollinia emarginata Schltdl. & Tree & $\mathrm{X}$ & $\mathrm{X}$ & & & & & & & 31403 \\
\hline \multirow[t]{2}{*}{ Apocynaceae } & $\begin{array}{l}\text { Aspidosperma cuspa (Kunth) S.F. } \\
\text { Blake ex Pittier }\end{array}$ & Tree & $\mathrm{X}$ & $\mathrm{X}$ & & $\mathrm{X}$ & & $\mathrm{X}$ & & $\mathrm{X}$ & 31405 \\
\hline & Aspidosperma sp. & Tree & & $\mathrm{X}$ & & $\mathrm{X}$ & & & & & 31406 \\
\hline Asteraceae & $\begin{array}{l}\text { Dasyphyllum brasiliense (Spreng.) } \\
\text { Cabrera* }\end{array}$ & Shrub & & & & & & & & & 31404 \\
\hline \multirow{3}{*}{ Bignoniaceae } & Jacaranda cuspidifolia Mart. & Tree & & & & & & $\mathrm{X}$ & & & $* *$ \\
\hline & $\begin{array}{l}\text { Tabebuia aurea (Silva Manso) Benth. } \\
\text { e Hook. f. ex S. Moore }\end{array}$ & Tree & $\mathrm{X}$ & & & & $\mathrm{X}$ & $\mathrm{X}$ & $\mathrm{X}$ & $\mathrm{X}$ & 31407 \\
\hline & Tabebuia roseoalba (Ridl.) Sandwith & Tree & & & & & & & & $\mathrm{X}$ & 31408 \\
\hline Burseraceae & $\begin{array}{l}\text { Protium heptaphyllum (Aubl.) } \\
\text { Marchand* }\end{array}$ & Tree & & & & & & & & & $* *$ \\
\hline Cannabaceae & Celtis spinosa Spreng. & Tree & $\mathrm{X}$ & & & & & & & & 31409 \\
\hline Celastraceae & Maytenus sp. & Tree & $\mathrm{X}$ & & & & & & & & 31410 \\
\hline Chrysobalanaceae & Hirtella sp.* & Tree & & & & & & & & & $* *$ \\
\hline \multirow{2}{*}{ Combretaceae } & Buchenavia tomentosa Eichler* & Tree & & & & & & & & & $* *$ \\
\hline & Terminalia argentea Mart. & Tree & & $\mathrm{X}$ & $\mathrm{X}$ & $\mathrm{X}$ & $\mathrm{X}$ & $\mathrm{X}$ & $\mathrm{X}$ & $\mathrm{X}$ & 31411 \\
\hline Clusiaceae & Kielmeyera coriacea Mart. e Zucc. & Tree & & & & & $\mathrm{X}$ & $\mathrm{X}$ & & & 31412 \\
\hline \multirow{2}{*}{ Dilleniaceae } & Curatella americana $\mathrm{L}$. & Tree & & & & & $\mathrm{X}$ & & $\mathrm{X}$ & $\mathrm{X}$ & 31413 \\
\hline & Davilla elliptica A. St.-Hil. & Tree & & & & & $\mathrm{X}$ & & & $\mathrm{X}$ & 31414 \\
\hline \multirow{2}{*}{ Erythroxylaceae } & $\begin{array}{l}\text { Erythroxylum cf. cuneifolium (Mart.) } \\
\text { O.E. Schulz }\end{array}$ & Shrub & $\mathrm{X}$ & & & $\mathrm{X}$ & & $\mathrm{X}$ & & $\mathrm{X}$ & 31415 \\
\hline & $\begin{array}{l}\text { Erythroxylum cf. suberosum A. St.- } \\
\text { Hil. }\end{array}$ & Tree & & $\mathrm{X}$ & $\mathrm{X}$ & $\mathrm{X}$ & $\mathrm{X}$ & $\mathrm{X}$ & $\mathrm{X}$ & $\mathrm{X}$ & 31416 \\
\hline \multirow{2}{*}{ Euphorbiaceae } & Acalypha villosa Jacq. & Shrub & $\mathrm{X}$ & $\mathrm{X}$ & & $\mathrm{X}$ & & & & & 31417 \\
\hline & Sebastiana brasiliensis Spreng. & Tree & $\mathrm{X}$ & & & $\mathrm{X}$ & & & & & 31418 \\
\hline Fabaceae & $\begin{array}{l}\text { Anadenanthera colubrina (Vell.) } \\
\text { Brenan }\end{array}$ & Tree & & & $\mathrm{X}$ & & $\mathrm{X}$ & & & $\mathrm{X}$ & 29181 \\
\hline
\end{tabular}


TABLE I (continuation)

\begin{tabular}{|c|c|c|c|c|c|c|c|c|c|c|c|}
\hline Family & Species & $\begin{array}{c}\text { Growth } \\
\text { habit }\end{array}$ & P1 & $\mathbf{P 2}$ & P3 & P4 & P5 & P6 & P7 & P8 & Voucher \\
\hline \multirow{9}{*}{ Fabaceae } & Bauhinia longifolia D. Dietr. & Shrub & & $\mathrm{X}$ & & $\mathrm{X}$ & & & & & 31419 \\
\hline & Bauhinia mollis (Bong.) D. Dietr. & Shrub & & & & & $\mathrm{X}$ & & & & 31420 \\
\hline & Bauhinia ungulata $\mathrm{L}$. & Tree & $\mathrm{X}$ & $\mathrm{X}$ & $\mathrm{X}$ & & & & & & 31421 \\
\hline & Bauhinia sp.1 & Tree & & & $\mathrm{X}$ & & & & $\mathrm{X}$ & & 31422 \\
\hline & Bauhinia sp.2 & Tree & & & & & $\mathrm{X}$ & & & & 31423 \\
\hline & Hymenaea martiana Hayne ${ }^{\star}$ & Shrub & & & & & & & & & $* *$ \\
\hline & Machaerium acutifolium Vogel ${ }^{\star}$ & Tree & & & & & & & & & ** \\
\hline & Pterogyne nitens Tul.* & Tree & & & & & & & & & ** \\
\hline & Sclerolobium sp.* & Tree & & & & & & & & & ** \\
\hline \multirow{3}{*}{ Lythraceae } & Adenaria floribunda Kunth* & Shrub & & & & & & & & & 31424 \\
\hline & Lafoensia pacari A. St.-Hil. & Tree & & & & $\mathrm{X}$ & & & $\mathrm{X}$ & & 31425 \\
\hline & Byrsonima sp. & Shrub & & & & & & & $\mathrm{X}$ & & 31426 \\
\hline \multirow[t]{4}{*}{ Malpighiaceae } & Byrsonima intermedia A. Juss. & Shrub & & & & & $\mathrm{X}$ & & & & 31427 \\
\hline & Mascagnia sp.* & Tree & & & & & & & & & $* *$ \\
\hline & Guazuma ulmifolia Lam. & Tree & & $\mathrm{X}$ & $\mathrm{X}$ & & & & & $\mathrm{X}$ & 31428 \\
\hline & $\begin{array}{l}\text { Helicteres lhotzkyana (Schott e Endl.) K. } \\
\text { Schum. }\end{array}$ & Tree & $\mathrm{X}$ & & $\mathrm{X}$ & $\mathrm{X}$ & $\mathrm{X}$ & & & & 31429 \\
\hline \multirow{5}{*}{ Malvaceae } & Luehea paniculata Mart.* & Tree & $\mathrm{X}$ & $\mathrm{X}$ & $\mathrm{X}$ & $\mathrm{X}$ & $\mathrm{X}$ & $\mathrm{X}$ & $\mathrm{X}$ & $\mathrm{X}$ & 31431 \\
\hline & Luehea sp. & Tree & & & $\mathrm{X}$ & & & & & & \\
\hline & $\begin{array}{l}\text { Pseudobombax cf. tomentosum (Mart. e } \\
\text { Zucc.) Robyns }\end{array}$ & Tree & $\mathrm{X}$ & & & & $\mathrm{X}$ & $\mathrm{X}$ & $\mathrm{X}$ & & 31432 \\
\hline & Sterculia striata A. St.-Hil. e Naudin* & Tree & & & & & & & & & $* *$ \\
\hline & Ficus insipida Willd. & Tree & & & & & & $\mathrm{X}$ & & & 31433 \\
\hline \multirow[t]{4}{*}{ Moraceae } & $\begin{array}{l}\text { Sorocea cf. bonplandii (Baill.) W.C. } \\
\text { Burger, Lanj. e Wess. Boer* }\end{array}$ & Tree & & & & & & & & & $* *$ \\
\hline & Campomanesia sp. & Tree & & & & & & & & $\mathrm{X}$ & 31435 \\
\hline & Myrcia guianensis (Aubl.) DC. & Shrub & & & & & $\mathrm{X}$ & & & $\mathrm{X}$ & 31436 \\
\hline & Myrcia laruotteana Cambess. & Tree & $\mathrm{X}$ & & $\mathrm{X}$ & & & & & & 31437 \\
\hline \multirow[t]{4}{*}{ Myrtaceae } & Myrcia tomentosa (Aubl.) DC. & Tree & $\mathrm{X}$ & $\mathrm{X}$ & & & $\mathrm{X}$ & & & $\mathrm{X}$ & 31438 \\
\hline & Myrcia torta DC. & Shrub & & & & & & & & & 31439 \\
\hline & Myrtaceae sp. 1 & Shrub & & & & & & & $\mathrm{X}$ & $\mathrm{X}$ & 31440 \\
\hline & Psidium guineense $\mathrm{Sw}$. & Tree & & & $\mathrm{X}$ & $\mathrm{X}$ & & & & & 31441 \\
\hline Nyctaginaceae & Guapira areolata (Heimerl) Lundell* & Tree & & & & & & & & & $* *$ \\
\hline Opiliaceae & $\begin{array}{l}\text { Agonandra brasiliensis Miers ex Benth. e } \\
\text { Hook. f.* }\end{array}$ & Tree & & & & & & & & & $* *$ \\
\hline Primulaceae & Rapanea gardneriana (A. DC.) Mez* & Shrub & & & & & & & & & 31430 \\
\hline Proteaceae & Roupala montana Aubl. & Tree & & & & & & & $\mathrm{X}$ & $\mathrm{X}$ & 31442 \\
\hline Rhamnaceae & Rhamnidium elaeocarpum Reissek & Tree & & & & $\mathrm{X}$ & & $\mathrm{X}$ & $\mathrm{X}$ & $\mathrm{X}$ & 31443 \\
\hline \multirow{4}{*}{ Rubiaceae } & Alibertia edulis (Rich.) A. Rich. ex DC. & Shrub & $\mathrm{X}$ & $\mathrm{X}$ & $\mathrm{X}$ & $\mathrm{X}$ & $\mathrm{X}$ & $\mathrm{X}$ & $\mathrm{X}$ & $\mathrm{X}$ & 31444 \\
\hline & Alibertia sp. & Tree & $\mathrm{X}$ & & & & & & & & $* *$ \\
\hline & Calycophyllum multiflorum Griseb.* & Tree & & & & & & & & & 31445 \\
\hline & Chomelia cf. pohliana Müll. Arg.* & Shrub & & & & & & & & & $* *$ \\
\hline
\end{tabular}


TABLE I (continuation)

\begin{tabular}{|c|c|c|c|c|c|c|c|c|c|c|c|}
\hline Family & Species & $\begin{array}{c}\text { Growth } \\
\text { habit }\end{array}$ & P1 & $\mathbf{P 2}$ & $\mathbf{P 3}$ & P4 & P5 & P6 & P7 & P8 & Voucher \\
\hline \multirow{2}{*}{ Rubiaceae } & Guettarda viburnoides Cham. e Schltdl. & Tree & & & $\mathrm{X}$ & $\mathrm{X}$ & & $\mathrm{X}$ & $\mathrm{X}$ & $\mathrm{X}$ & 31446 \\
\hline & Randia armata (Sw.) DC. & Tree & & & $\mathrm{X}$ & & & & & & 31447 \\
\hline \multirow{2}{*}{ Salicaceae } & Casearia rupestris Eichler & Tree & $\mathrm{X}$ & $\mathrm{X}$ & $\mathrm{X}$ & & & & & & 31448 \\
\hline & Xylosma venosa N.E. Br.* & Tree & & & & & & & & & 31449 \\
\hline \multirow{3}{*}{ Sapindaceae } & Cupania castaneifolia Mart.* & Tree & & & & & & & & & $* *$ \\
\hline & Dilodendron bipinnatum Radlk. & Shrub & & $\mathrm{X}$ & & & & & & $\mathrm{X}$ & 31450 \\
\hline & Magonia pubescens A. St.-Hil.* & Tree & & & & & & & & & $* *$ \\
\hline \multirow[t]{2}{*}{ Sapotaceae } & $\begin{array}{l}\text { Chrysophyllum marginatum (Hook. e Arn.) } \\
\text { Radlk. }\end{array}$ & Tree & & & & $\mathrm{X}$ & & & & $\mathrm{X}$ & 31451 \\
\hline & Pouteria gardneri (Mart. e Miq.) Baehni & Tree & $\mathrm{X}$ & & & & & & & & 31452 \\
\hline \multirow{2}{*}{ Vochysiaceae } & Callisthene fasciculata Mart. & Tree & $\mathrm{X}$ & $\mathrm{X}$ & $\mathrm{X}$ & $\mathrm{X}$ & $\mathrm{X}$ & $\mathrm{X}$ & $\mathrm{X}$ & $\mathrm{X}$ & 31453 \\
\hline & Qualea parviflora Mart. & Tree & $\mathrm{X}$ & $\mathrm{X}$ & X & X & X & $\mathrm{X}$ & $\mathrm{X}$ & $\mathrm{X}$ & 31454 \\
\hline
\end{tabular}

Based on the floristic plot data, group mean (UPGMA) revealed the presence of two plant groupings (see Figure 3). Grouping 1 (A1) was formed by plots 1, 2, 3 and 4 and grouping 2 (A2) by plots 5, 6, 7 and 8 . Both A1 and A2 comprised 38 species, 16 of which were exclusive to each (see Table I); the Sørensen index yielded a 0.71 cophenetic correlation coefficient.

Ordination through Principal Component Analysis (PCA) of the soil of the eight plots analyzed (see Figure 4) presented a cumulative variance of $59.0 \%$, and eigenvalues of axes 1 and 2 were 2.11 and 2.56 , respectively. These results were similar to those of the floristic groupings (see Figure 3), the only difference being the distance between plots 4 and 5 . Considering the groupings found through floristic similarity, A1 and A2 were practically equal for fertility $(p=0.74)$. Among the floristic groupings, phosphorus $(\mathrm{P})(\mathrm{p}<0.01)$, copper $(\mathrm{Cu})(\mathrm{p}<0.01)$ and zinc $(\mathrm{Zn})(\mathrm{p}=0.01)$ differ significantly (see Table III).

During the period of the study, 54 species were monitored for reproductive phenology at community level: 25 flowered and 52 fruiting (see Table II; see Figure 2). As for the 38 species found in each area, 19 and 22 species were observed in flower in A1 and A2, respectively (see
Table II). The difference between both groupings was not significant $(U=53.5 ; p=0.28)$. Flowering was uniform during the analyzed year for both groupings A1 and A2; mean angles were $268.6^{\circ}$ (A1+A2), $249.0^{\circ}$ (A1), corresponding to the month of September, and $236.8^{\circ}$ (A2), corresponding to August (see Table V).

The number of flowering species was negatively related to rainfall in $\mathrm{A} 2$ and presented no correlations to temperature or day length in either grouping (see Table IV).

Fructification occurred in 35 species in A1 and in 36 species in A2 without any significant difference $(U=59.5 ; p=0.47)$ and was moderately seasonal for both groupings $\mathrm{A} 1$ and $\mathrm{A} 2$; mean angles were $10.1^{\circ}, 11.4^{\circ}$ and $11.9^{\circ}$, respectively, corresponding to January for all (see Table V). Fructification was explained by the abiotic factors analyzed (see Table IV).

With regards to dispersal, zoochory predominated in 32 species, followed by anemo- and autochory with 13 and 11 species, respectively. Zoochory also prevailed in both groupings (see Table II), and there were no differences between the distribution of dispersal syndromes $\left(\mathrm{x}^{2}=1.173 ; \mathrm{p}=0.56\right)$. All syndromes were seasonal throughout the year in both groupings. Mean 


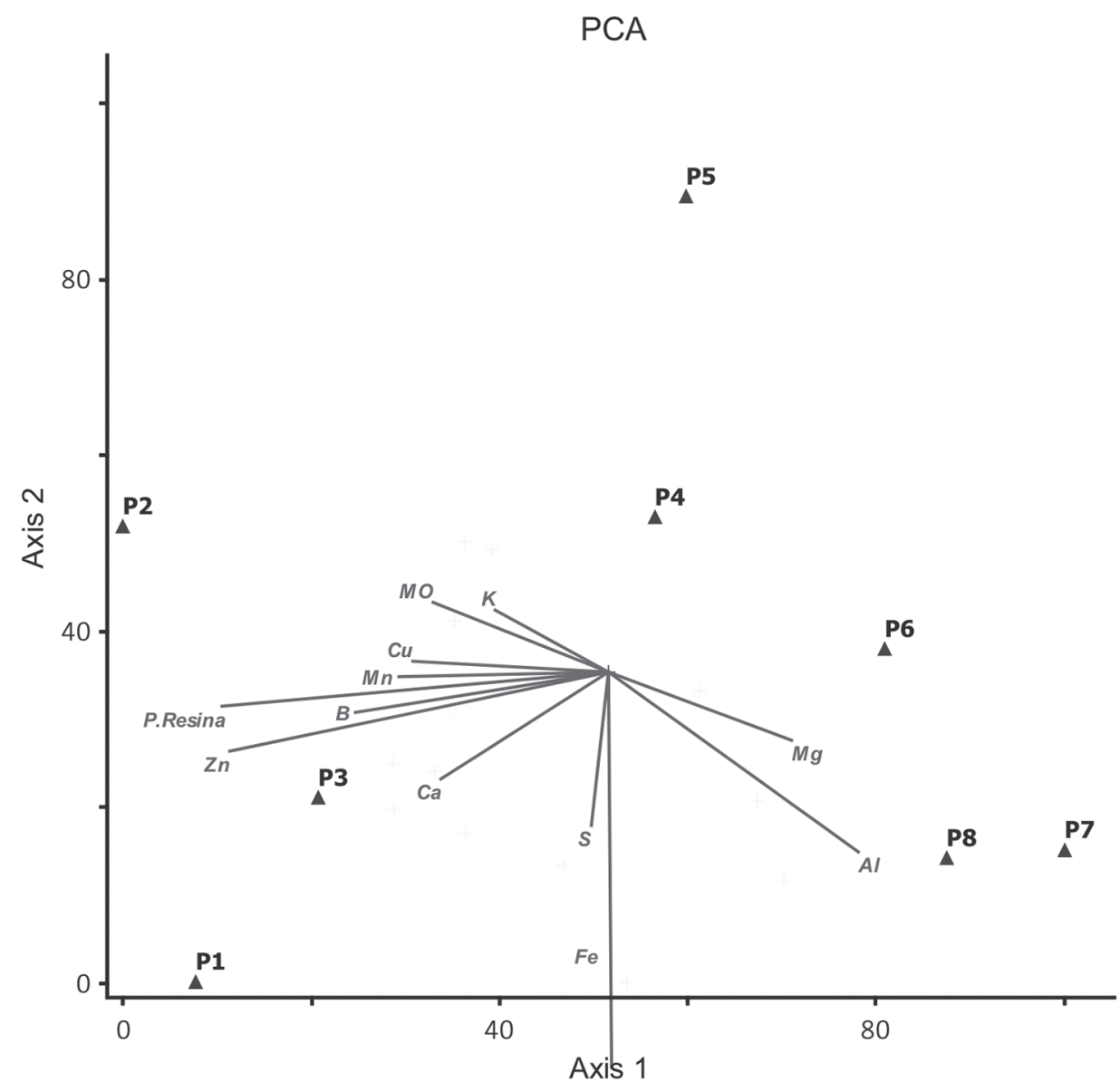

Figure 3 - Dendrogram produced through group mean (UPGMA) for the plots analyzed through the Sørensen similarity index in the floristic study on an escarpment slope in the Serra de Maracaju, Mato Grosso do Sul, Brazil (cophenetic correlation $=0.71$ ).

\section{TABLE II}

List of families and species, dispersal syndromes, flowering and fructification in the plots of an escarpment slope of the Serra de Maracaju, Mato Grosso do Sul, Brazil. $($ Jan = January, Feb = February, Mar $=$ March, Apr = April, May = May, Jun = June, Jul = July, Aug = August, Sep = September, Oct $=$ October, Nov $=$ November, Dec $=$ December; Ane $=$ Anemochory, Zoo $=$ Zoochory, Aut $=$ Autochory; $\square$ Flowering; $\square$ Fructification; $\square$ Flowering and Fructification. 2009 and 2010 represent the reproductive phenophases.

\begin{tabular}{|c|c|c|c|c|c|c|c|c|c|c|c|c|c|c|}
\hline Family & Species & $\begin{array}{l}\text { Dispersal } \\
\text { syndromes }\end{array}$ & 2009 & 2010 & & & & & & & & & & \\
\hline & & & Dec & Jan & Feb & Mar & Apr & May & Jun & Jul & Aug & Sep & Oct & Nov \\
\hline \multirow[t]{2}{*}{ Anacardiaceae } & $\begin{array}{l}\text { Astronium fraxinifolium } \\
\text { Schott ex Spreng. }\end{array}$ & Ane & & & & & & & & & & & & \\
\hline & Lithraea molleoides Engl. & Zoo & & & & & & & & & & & & \\
\hline \multirow{3}{*}{ Annonaceae } & $\begin{array}{l}\text { Annona cornifolia A. } \\
\text { St.-Hil. }\end{array}$ & Zoo & & & & & & & & & & & & \\
\hline & Annona crassiflora Mart. & Zoo & & & & & & & & & & & & \\
\hline & $\begin{array}{l}\text { Rollinia emarginata } \\
\text { Schltdl. }\end{array}$ & Zoo & & & & & & & & & & & & \\
\hline
\end{tabular}


TABLE II (continuation)

\begin{tabular}{|c|c|c|c|c|c|c|c|c|c|c|c|c|c|c|}
\hline Family & Species & $\begin{array}{l}\text { Dispersal } \\
\text { syndromes }\end{array}$ & 2009 & 2010 & & & & & & & & & & \\
\hline & & & Dec & Jan & Feb & Mar & Apr & May & Jun & Jul & Aug & Sep & Oct & Nov \\
\hline \multirow[t]{2}{*}{ Apocynaceae } & $\begin{array}{l}\text { Aspidosperma cuspa } \\
\text { (Kunth) S.F. Blake ex } \\
\text { Pittier }\end{array}$ & Ane & & & & & & & & & & & & \\
\hline & Aspidosperma sp. & Ane & & & & & & & & & & & & \\
\hline \multirow{2}{*}{ Bignoniaceae } & $\begin{array}{l}\text { Tabebuia aurea (Silva } \\
\text { Manso) Benth. e Hook. } \\
\text { f. ex S. Moore }\end{array}$ & Ane & & & & & & & & & & & & \\
\hline & $\begin{array}{l}\text { Tabebuia roseoalba } \\
\text { (Ridl.) Sandwith }\end{array}$ & Ane & & & & & & & & & & & & \\
\hline Cannabaceae & Celtis spinosa Spreng. & Zoo & & & & & & & & & & & & \\
\hline Celastraceae & Maytenus sp. & Zoo & & & & & & & & & & & & \\
\hline Combretaceae & $\begin{array}{l}\text { Terminalia argentea } \\
\text { Mart. }\end{array}$ & Ane & & & & & & & & & & & & \\
\hline Clusiaceae & $\begin{array}{l}\text { Kielmeyera coriacea } \\
\text { Mart. e Zucc. }\end{array}$ & Ane & & & & & & & & & & & & \\
\hline \multirow[b]{2}{*}{ Dilleniaceae } & Curatella americana $\mathrm{L}$. & Zoo & & & & & & & & & & & & \\
\hline & $\begin{array}{l}\text { Davilla elliptica A. } \\
\text { St.-Hil. }\end{array}$ & Zoo & & & & & & & & & & & & \\
\hline \multirow[t]{2}{*}{ Bignoniaceae } & $\begin{array}{l}\text { Tabebuia aurea (Silva } \\
\text { Manso) Benth. e Hook. } \\
\text { f. ex S. Moore }\end{array}$ & Ane & & & & & & & & & & & & \\
\hline & $\begin{array}{l}\text { Tabebuia roseoalba } \\
\text { (Ridl.) Sandwith }\end{array}$ & Ane & & & & & & & & & & & & \\
\hline Cannabaceae & Celtis spinosa Spreng. & Zoo & & & & & & & & & & & & \\
\hline Celastraceae & Maytenus sp. & Zoo & & & & & & & & & & & & \\
\hline Combretaceae & $\begin{array}{l}\text { Terminalia argentea } \\
\text { Mart. }\end{array}$ & Ane & & & & & & & & & & & & \\
\hline Clusiaceae & $\begin{array}{l}\text { Kielmeyera coriacea } \\
\text { Mart. e Zucc. }\end{array}$ & Ane & & & & & & & & & & & & \\
\hline \multirow[b]{2}{*}{ Dilleniaceae } & Curatella americana L. & Zoo & & & & & & & & & & & & \\
\hline & $\begin{array}{l}\text { Davilla elliptica A. } \\
\text { St.-Hil. }\end{array}$ & Zoo & & & & & & & & & & & & \\
\hline \multirow[t]{2}{*}{ Erythroxylaceae } & $\begin{array}{l}\text { Erythroxylum cf. } \\
\text { cuneifolium (Mart.) O.E. } \\
\text { Schulz }\end{array}$ & Zoo & & & & & & & & & & & & \\
\hline & $\begin{array}{l}\text { Erythroxylum cf. } \\
\text { suberosum A. St.-Hil. }\end{array}$ & Zoo & & & & & & & & & & & & \\
\hline Bignoniaceae & $\begin{array}{l}\text { Jacaranda cuspidifolia } \\
\text { Mart. }\end{array}$ & Ane & & & & & & & & & & & & \\
\hline \multirow[b]{2}{*}{ Euphorbiaceae } & Acalypha villosa Jacq. & Aut & & & & & & & & & & & & \\
\hline & $\begin{array}{l}\text { Sebastiana brasiliensis } \\
\text { Spreng. }\end{array}$ & Aut & & & & & & & & & & & & \\
\hline \multirow{6}{*}{ Fabaceae } & $\begin{array}{l}\text { Anadenanthera } \\
\text { colubrina (Vell.) Brenan }\end{array}$ & Aut & & & & & & & & & & & & \\
\hline & $\begin{array}{l}\text { Bauhinia longifolia D. } \\
\text { Dietr. }\end{array}$ & Aut & & & & & & & & & & & & \\
\hline & $\begin{array}{l}\text { Bauhinia mollis (Bong.) } \\
\text { D. Dietr. }\end{array}$ & Aut & & & & & & & & & & & & \\
\hline & Bauhinia ungulata L. & Aut & & & & & & & & & & & & \\
\hline & Bauhinia sp.1 & Aut & & & & & & & & & & & & \\
\hline & Bauhinia sp.2 & Aut & & & & & & & & & & & & \\
\hline
\end{tabular}


TABLE II (continuation)

\begin{tabular}{|c|c|c|c|c|c|c|c|c|c|c|c|c|c|c|}
\hline Family & Species & $\begin{array}{c}\text { Dispersal } \\
\text { syndromes }\end{array}$ & 2009 & 2010 & & & & & & & & & & \\
\hline & & & Dec & Jan & Feb & Mar & Apr & May & Jun & Jul & Aug & Sep & Oct & Nov \\
\hline Lythraceae & Lafoensia pacari A. St.-Hil. & Ane & & & & & & & & & & & & \\
\hline \multirow[b]{2}{*}{ Malpighiaceae } & Byrsonima sp. & Zoo & & & & & & & & & & & & \\
\hline & $\begin{array}{l}\text { Byrsonima intermedia A. } \\
\text { Juss. }\end{array}$ & Zoo & & & & & & & & & & & & \\
\hline \multirow[b]{2}{*}{ Malvaceae } & Guazuma ulmifolia Lam. & Zoo & & & & & & & & & & & & \\
\hline & $\begin{array}{l}\text { Helicteres lhotzkyana } \\
\text { (Schott e Endl.) K. Schum. }\end{array}$ & Aut & & & & & & & & & & & & \\
\hline \multirow[b]{3}{*}{ Malvaceae } & Luehea paniculata Mart. & Ane & & & & & & & & & & & & \\
\hline & Luehea sp. & Ane & & & & & & & & & & & & \\
\hline & $\begin{array}{l}\text { Pseudobombax cf. } \\
\text { tomentosum (Mart. e Zucc.) } \\
\text { Robyns }\end{array}$ & Ane & & & & & & & & & & & & \\
\hline Moraceae & Ficus insipida Willd. & Zoo & & & & & & & & & & & & \\
\hline \multirow{7}{*}{ Myrtaceae } & Campomanesia sp. & Zoo & & & & & & & & & & & & \\
\hline & $\begin{array}{l}\text { Myrcia guianensis (Aubl.) } \\
\text { DC. }\end{array}$ & Zoo & & & & & & & & & & & & \\
\hline & $\begin{array}{l}\text { Myrcia laruotteana } \\
\text { Cambess. }\end{array}$ & Zoo & & & & & & & & & & & & \\
\hline & $\begin{array}{l}\text { Myrcia tomentosa (Aubl.) } \\
\text { DC. }\end{array}$ & Zoo & & & & & & & & & & & & \\
\hline & Myrcia torta DC. & Zoo & & & & & & & & & & & & \\
\hline & Myrtaceae sp. 1 & Zoo & & & & & & & & & & & & \\
\hline & Psidium guineense $\mathrm{Sw}$. & Zoo & & & & & & & & & & & & \\
\hline Proteaceae & Roupala montana Aubl. & Ane & & & & & & & & & & & & \\
\hline Rhamnaceae & $\begin{array}{l}\text { Rhamnidium elaeocarpum } \\
\text { Reissek }\end{array}$ & Zoo & & & & & & & & & & & & \\
\hline \multirow{4}{*}{ Rubiaceae } & $\begin{array}{l}\text { Alibertia edulis (Rich.) A. } \\
\text { Rich. ex DC. }\end{array}$ & Zoo & & & & & & & & & & & & \\
\hline & Alibertia sp. & Zoo & & & & & & & & & & & & \\
\hline & $\begin{array}{l}\text { Guettarda } \\
\text { viburnoides Cham. e Schltdl. }\end{array}$ & Zoo & & & & & & & & & & & & \\
\hline & Randia armata (Sw.) DC. & Zoo & & & & & & & & & & & & \\
\hline \multirow[b]{2}{*}{ Salicaceae } & Casearia rupestris Eichler & Zoo & & & & & & & & & & & & \\
\hline & $\begin{array}{l}\text { Dilodendron bipinnatum } \\
\text { Radlk. }\end{array}$ & Ane & & & & & & & & & & & & \\
\hline \multirow{2}{*}{ Sapotaceae } & $\begin{array}{l}\text { Chrysophyllum marginatum } \\
\text { (Hook. e Arn.) Radlk. }\end{array}$ & Zoo & & & & & & & & & & & & \\
\hline & $\begin{array}{l}\text { Pouteria gardneri (Mart. e } \\
\text { Miq.) Baehni }\end{array}$ & Zoo & & & & & & & & & & & & \\
\hline \multirow{2}{*}{ Vochysiaceae } & Callisthene fasciculata Mart. & Ane & & & & & & & & & & & & \\
\hline & Qualea parviflora Mart. & Ane & & & & & & & & & & & & \\
\hline
\end{tabular}

angles were: $(\mathrm{A} 1+\mathrm{A} 2)=17.9^{\circ}, \quad \mathrm{A} 1=17.3$ and $\mathrm{A} 2=22.7$, corresponding to January, for zoochory; $(\mathrm{A} 1+\mathrm{A} 2)=267.6^{\circ}, \quad \mathrm{A} 1=263.1^{\circ}, \quad$ corresponding to September, and $\mathrm{A} 2=32.6^{\circ}$ corresponding to February, for anemochory; and finally, $(\mathrm{A} 1+\mathrm{A} 2)=$ $32.6^{\circ}$ and $\mathrm{A} 2=45.0^{\circ}$ corresponding to February, and $\mathrm{A} 1=29.6^{\circ}$, corresponding to January, for autochory (see Table V). These months represent the largest production for each syndrome and suggest that they prevail in the rainy period. 


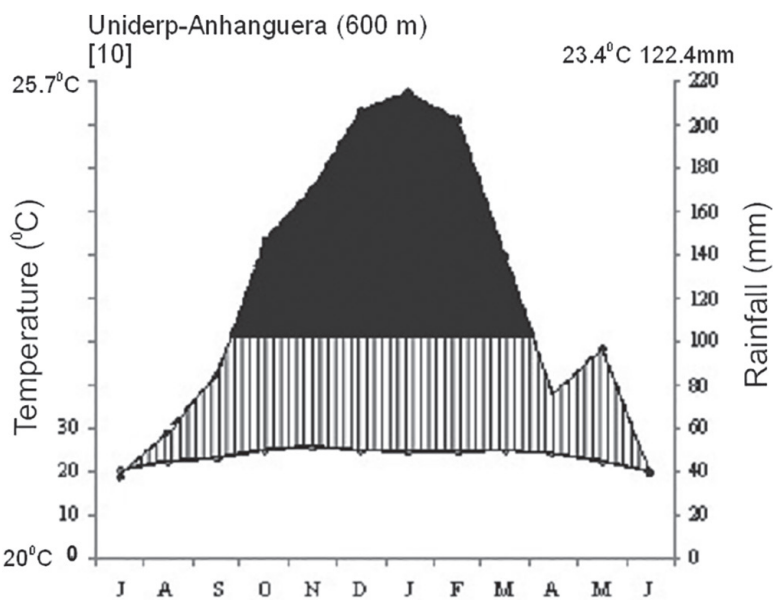

Figure 4 - Ordination through Principal Component Analysis (PCA) of the eight plots analyzed on an escarpment slope of the Serra de Maracaju, Mato Grosso do Sul, Brazil. The percentage of cumulative variance explained by the two axes is 39.34 and 59\%. (P.Resin = phosphorus, M.O.= organic matter, $\mathrm{K}=$ potassium, $\mathrm{Ca}=$ calcium, $\mathrm{Mg}=$ magnesium, $\mathrm{Al}=$ aluminum, $\mathrm{S}=$ sulfur, $\mathrm{B}=$ boron, $\mathrm{Cu}=$ copper, $\mathrm{Fe}=$ iron, $\mathrm{Zn}=$ zinc, $\mathrm{Mn}=$ manganese).

\section{DISCUSSION}

The high specific richness of Fabaceae and Myrtaceae in western and southeastern Brazil (B.G. Fina, unpublished data, Ruggiero et al. 2002) consolidates the link between the Cerrado and the Atlantic Forest (Pinto and Oliveira-Filho 1999). The present study showed that Fabaceae

\section{TABLE III}

Mean and standard deviation from mean (SDM) of soil fertility at 0-20 cm depth in the eight plots sampled on an escarpment slope in the Serra de Maracaju, Mato Grosso do Sul, Brazil. Elements S, B, Cu, Fe, Mn and $\mathrm{Zn}$ in $\mathrm{mg} / \mathrm{dm}^{3}$, organic matter (M.O.) in $\mathrm{g} / \mathrm{dm}^{3}$. Except for pH, the other elements and parameters are in mmolc/ $\mathrm{dm}^{3} . \mathrm{V} \%=$ percentage of base saturation; $\mathrm{BS}=$ base sum $(\mathrm{K}+\mathrm{Ca}+\mathrm{Mg}) ; \mathrm{H}+\mathrm{Al}=$ potential acidity $\mathbf{C T C}=$ Cation Exchange Capacity $(\mathrm{BS}+\mathrm{H}+\mathrm{Al})$. $(\mathrm{A} 1=$ plots $1,2,3,4 ; \mathrm{A} 2=$ plots 5,6,7,8; P.Resin = phosphor; M.O.= organic matter;

$\mathrm{K}=$ potassium; $\mathbf{C a}=$ calcium; $\mathbf{M g}=$ magnesium; $\mathbf{A l}=$ aluminum; $\mathrm{S}=$ sulfur; $\mathrm{B}=$ boron; $\mathrm{Cu}=$ copper; $\mathrm{Fe}=$ iron; $\mathrm{Zn}=$ zinc; $\mathrm{Mn}=$ manganese).

\begin{tabular}{|c|c|c|c|}
\hline \multicolumn{4}{|c|}{$\mathrm{X} \pm \mathrm{DPM}$} \\
\hline & A1 & A2 & $\mathrm{P}$ \\
\hline P Resin & $210.25 \pm 65.98$ & $22.29 \pm 22.29$ & $<0.01$ \\
\hline M.O. & $72.50 \pm 14.91$ & $62.50 \pm 14.84$ & 0.38 \\
\hline $\mathrm{pH}$ & $5.68 \pm 0.15$ & $5.78 \pm 0.17$ & 0.41 \\
\hline $\mathrm{K}$ & $2.35 \pm 0.78$ & $1.83 \pm 0.97$ & 0.43 \\
\hline $\mathrm{Ca}$ & $253.00 \pm 29.02$ & $213.50 \pm 36.36$ & 0.14 \\
\hline $\mathrm{Mg}$ & $70.75 \pm 5.19$ & $87.25 \pm 18.79$ & 0.14 \\
\hline $\mathrm{H}+\mathrm{Al}$ & $39.75 \pm 5.91$ & $35.00 \pm 3.92$ & 0.23 \\
\hline $\mathrm{Al}$ & $0.63 \pm 0.15$ & $0.80 \pm 0.20$ & 0.21 \\
\hline SB & $326.10 \pm 31.30$ & $302.58 \pm 49.96$ & 0.46 \\
\hline CTC & $365.85 \pm 31.97$ & $337.58 \pm 47.09$ & 0.36 \\
\hline V & $89.00 \pm 1.63$ & $89.5 \pm 2.38$ & 0.74 \\
\hline $\mathrm{S}$ & $10.50 \pm 5.57$ & $9.00 \pm 2.83$ & 0.65 \\
\hline B & $0.89 \pm 0.47$ & $0.49 \pm 0.16$ & 0.16 \\
\hline $\mathrm{Cu}$ & $10.73 \pm 1.38$ & $6.95 \pm 2.91$ & $<0.01$ \\
\hline $\mathrm{Fe}$ & $101.00 \pm 24.86$ & $96.75 \pm 24.12$ & 0.81 \\
\hline $\mathrm{Mn}$ & $285.75 \pm 90.17$ & $193.50 \pm 57.39$ & 0.14 \\
\hline $\mathrm{Zn}$ & $2.20 \pm 0.61$ & $1.08 \pm 0.24$ & 0.01 \\
\hline
\end{tabular}

TABLE IV

Correlation between abiotic factors and reproductive phenology of trees and bushes on an escarpment slope in the Serra de Maracaju, Mato Grosso do Sul, Brazil. (A1= plots 1,2,3 and 4; $\mathrm{A} 2=$ plots 5,6,7 and 8; $r=$ result of Spearman analysis; $p=$ significance). ${ }^{1}$ Study period, ${ }^{2} 10$ years.

\begin{tabular}{|c|c|c|c|c|c|c|}
\hline & $\mathrm{A} 1+\mathrm{A} 2$ & $\mathrm{p}$ & A1 & $\mathrm{p}$ & $\mathrm{A} 2$ & $\mathrm{P}$ \\
\hline Flowering X Rainfall $^{1}$ & $\mathrm{r}=-0.53$ & 0.08 & $\mathrm{r}=0.59$ & 0.46 & $\mathrm{r}=-0.71$ & 0.01 \\
\hline Flowering X Rainfall $^{2}$ & $r=-0.15$ & 0.64 & $\mathrm{r}=-0.40$ & 0.19 & $\mathrm{r}=-0.59$ & 0.05 \\
\hline Flowering $\mathrm{x}$ Day length ${ }^{1}$ & $\mathrm{r}=-0.23$ & 0.47 & $\mathrm{r}=-0.47$ & 0.13 & $\mathrm{r}=-0.32$ & 0.31 \\
\hline Flowering $x$ Mean temperature ${ }^{1}$ & $\mathrm{r}=0.05$ & 0.87 & $r=-0.46$ & 0.13 & $r=-0.46$ & 0.13 \\
\hline Flowering x Mean temperature ${ }^{2}$ & $\mathrm{r}=-0.56$ & 0.06 & $r=-0.24$ & 0.44 & $\mathrm{r}=-0.24$ & 0.44 \\
\hline Fructification X Rainfall $^{1}$ & $\mathrm{r}=0.76$ & $<0.01$ & $\mathrm{r}=0.78$ & $<0.01$ & $\mathrm{r}=0.80$ & $<0.01$ \\
\hline Fructification X Rainfall $^{2}$ & $\mathrm{r}=0.73$ & $<0.01$ & $\mathrm{r}=0.68$ & 0.02 & $\mathrm{r}=0.62$ & 0.03 \\
\hline Fructification X Day length $^{1}$ & $\mathrm{r}=0.87$ & $<0.01$ & $\mathrm{r}=0.89$ & $<0.01$ & $\mathrm{r}=0.9$ & $<0.01$ \\
\hline Fructification X Mean temperature $^{1}$ & $\mathrm{r}=0.74$ & $<0.01$ & $\mathrm{r}=0.68$ & 0.02 & $\mathrm{r}=0.63$ & 0.03 \\
\hline Fructification X Mean temperature $^{2}$ & $\mathrm{r}=0.89$ & $<0.01$ & $\mathrm{r}=0.88$ & $<0.01$ & $\mathrm{r}=0.79$ & $<0.01$ \\
\hline
\end{tabular}


TABLE V

Circular analysis of the reproductive phenology of number of trees and bush species on an escarpment slope in the Serra de Maracaju, Mato Grosso do Sul, Brazil. (A1= plots 1,2,3 and 4; $A 2=$ plots 5,6,7 and $8 ; z=$ result of circular analysis; $p=$ significance).

\begin{tabular}{ccccccc}
\hline & $\mathrm{A} 1+\mathrm{A} 2$ & $\mathrm{p}$ & $\mathrm{A} 1$ & $\mathrm{p}$ & $\mathrm{A} 2$ & $\mathrm{P}$ \\
\hline Flowering & $\mathrm{Z}=7.96 \mathrm{E}-01$ & 0.45 & $\mathrm{Z}=1.40$ & 0.25 & $\mathrm{Z}=1.82$ & 0.16 \\
Fructification & $\mathrm{Z}=24.43$ & $2.47 \mathrm{E}-11$ & $\mathrm{Z}=25.89$ & $5.73 \mathrm{E}-12$ & $\mathrm{Z}=18.61$ & $8.29 \mathrm{E}-09$ \\
Zoochory & $\mathrm{Z}=59.6$ & 0 & $\mathrm{Z}=73.2$ & 0 & $\mathrm{Z}=48.3$ & 0 \\
Anemochory & $\mathrm{Z}=7.4$ & $5.94 \mathrm{E}-04$ & $\mathrm{Z}=8.7$ & $1.61 \mathrm{E}-04$ & $\mathrm{Z}=9.9$ & $4.79 \mathrm{E}-05$ \\
Autochory & $\mathrm{Z}=101.0$ & 0 & $\mathrm{Z}=145.2$ & 0 & $\mathrm{Z}=107.8$ & 0 \\
\hline
\end{tabular}

also prevails in environments with different, albeit close, formations, as observed by Gentry (1993) in neotropical environments. Malvaceae, the third most representative family in this study, is also reported among the main families occurring in the Serra de Maracaju in other localities (Ramos and Sartori 2013), while, according to Heywood et al. (2007), this family is common in savanna, "scrub" and forest edges.

Grouping 1 (A1) corresponds to a remnant of seasonal deciduous forest characterized by the occurrence of the same species, significant levels of leaf deciduousness in the dry period, according to field observations, and eutrophic soil. The presence of characteristic species of dry forests as Sebastiana brasiliensis, Bauhinia ungulata, Rollinia emarginata, Randia armata and Casearia rupestris is reported in other studies on similar formations (Nascimento et al. 2004, Salis et al. 2004, G.A. Damasceno-Junior, unpublished data, Pott et al. 2011). Grouping 2 (A2) here considered as cerradão shows characteristic species such as Curatella americana, Jacaranda cuspidifolia Annona crassiflora, Byrsonima intermedia, Davilla elliptica, Roupala montana. Our results are in agreement with previous studies such as Ribeiro and Walter (2008), and Ratter et al. (2006). Curatella americana is common in savannas from Central America to southern Brazil, and the presence of Roupala montana indicates soil fertility (Ratter et al. 2006).

Soil fertility analysis with a $\mathrm{pH}$ between 5.5 and 6.0, base saturation higher than 50\% and rugged landscapes, suggest eutrophic soil, according to the parameters listed by Ratter et al. (2003) and Reatto et al. (2008). The high content of phosphorus, zinc and copper available in A1 together with deeper soils reinforce the fact that it is a deciduous forest, according to Eiten (1982). A2 is a eutrophic cerradão since its $\mathrm{pH}$ is higher than expected dystrophic Cerrado in central Brazil, where it usually varies between 4.0 and 4.8 (Ratter et al. 1978). In addition, such plant formations prevail in basalt formations (Reatto et al. 2008), which is the case of the Serra de Maracaju (Martins 2003). Deciduous forest with fertile soil interspersed with Cerrado (cerradão) could have resulted from dry climate episodes during the Pleistocene, as described by Prado (2000) for South American areas. These formations in the Serra de Maracaju would have acted as corridors for species since they can shelter those common to other plant formations, such as the thorn forest (Chaco) and dry forests (Salis et al. 2004).

The uniform flowering and the negative correlation between flowering and rain, day length and mean temperature in the area of study can be explained by the fact that the soil contains water all year round, since it is interspersed with streams and springs in addition to rocky outcrops that possibly favor water retention. This pattern suggests that the tree and shrub species do not depend directly on rain to flower and probably rely on their deep roots to maintain their water supply (Sarmiento and Monasterio 1983, Batalha and Martins 2004). 
The moderately seasonal fructification and positive correlation between fructification and average rainfalls stresses the importance of favorable water conditions to yield fleshy fruits since, during the rainy season, easily acess to water contributes to fruit development (Marco and Paéz 2002); the positive correlation between fructification and both day length and mean temperature was expected in this seasonal formation (Morellato et al. 1990, Morellato et al. 2000).

The preponderance of zoochory followed by anemochory and autochory has already been reported in semi-deciduous forest and semideciduous altitude forests (Morellato and LeitãoFilho 1992, Kinoshita et al. 2006), in Cerrado (Batalha and Martins 2004), and it also prevails in tropical forests (Fleming 1979). The period when most species blossomed and fruited, independently of their respective dispersal modes, coincided with that of studies in Cerrado formations of west-central Brazil (Batalha and Martins 2004) and in semi-deciduous altitude forests and semideciduous forests of the southeastern region of Brazil (Morellato and Leitão-Filho 1992).

The intense deciduousness of some species observed during a given period of the year apparently suggests that the area of the Serra de Maracaju studied here is constituted by a one and only plant formation. Nevertheless, two adjacent phytophysiognomies, seasonal forest and cerradão, could be distinguished through floristic composition and soil fertility. These must be further analyzed both to confirm this classification and to conduct other studies on the slopes of this serra. The set formed by given tree and shrub species suggests that this portion of the Serra de Maracaju is floristically linked to the Atlantic Domain (dry forests) and the Cerrado (cerradão), a biogeographical aspect that should be further explored in future studies.

The correlations between reproductive phenophases and the abiotic variables did not differ between deciduous forest and cerradão. The phenological pattern obtained for flowering differed from what was expected in seasonal environments. This is possibly related to the specificities of the plant communities assessed and their evolutionary history. The preponderance of zoochory highlights the importance of animal-plant interaction to maintain the studied community. Nevertheless, abiotic syndromes do play a crucial role in the Serra de Maracaju formed by many slopes that create physical barriers to biotic agents.

\section{ACKNOWLEDGMENTS}

The first author wishes to thank the Fundação de Apoio ao Desenvolvimento do Ensino, Ciência e Tecnologia do Estado de Mato Grosso do Sul (FUNDECT) for the fellowship granted, as well as her colleagues and friends who helped her during field collection, and the Pró-Reitoria de Pesquisa e Pós-Graduação (PROPP - Dean for research and post-graduation) and the Program in Vegetal Biology for their logistical support.

\section{RESUMO}

Fatores ambientais, tais como temperatura, solo, fotoperíodo e precipitação podem determinar o ambiente físico favorecendo a ocorrência de espécies e interferir no período reprodutivo das plantas. Este trabalho foi realizado através de excursões mensais para uma encosta da Serra de Maracaju, entre dezembro de 2009 e novembro de 2010. As amostras foram coletadas em oito parcelas, $25 \times 50$ $\mathrm{m}$, posicionado transversalmente ao declive, totalizando 1 ha amostrado. A riqueza florística desta encosta incluiu 79 espécies distribuídas em 58 gêneros e 31 famílias. A análise de similaridade florística e a análise de Componentes Principais mostraram a ocorrência de duas formações vegetais na encosta: floresta estacional decidual e cerradão no solo eutrófico da encosta. Floração e frutificação ocorreram na estação chuvosa; correlação com a precipitação, temperatura média e do fotoperíodo foram negativas para a floração e positiva para a frutificação. Árvores e arbustos apresentaram floração uniforme e uma frutificação moderadamente 
sazonal, que é inesperado em ambientes sazonais. Nós discutimos as implicações dos padrões encontrados e fizemos comparações com outros estudos.

Palavras-chave: Cerradão, comunidade, floresta decídua, solo eutrófico.

\section{REFERENCES}

APG III - ANGIOSPERM PHYLOGENY GROUP. 2009. An update of the Angiosperm Phylogeny Group classification for the orders and families of flowering plants: APG III. Bot Soc Linn J 161: 105-121.

BATALHA MA AND MANTOVANI W. 2000. Reproductive phenological patterns of Cerrado plant species at the Pé-de Gigante Reserve (Santa Rita do Passa Quatro, SP, Brazil): a comparation between the herbaceus and woody floras. Rev Bras Biol 60: 129-145.

BATALHA MAAND MARTINS FR. 2004. Reproductive phenology of the Cerrado plant community in Emas National Park (central Brazil). Aust J Bot 52: 149-161.

CAIN SA AND CASTRO GMO. 1959. Manual of vegetation analysis. New York: Hafner. xvii +325 p.

EITEN G. 1982. Brazilian 'Savannas'. In: HUNTLEY BJ AND WALKER BH (Eds), Ecology of tropical savannas. Germany: Springer Verlag, Ecological Studies 42: 25-47.

FELFILI JM, REZENDE AV AND SILVA-JÚNIOR MC. 2007. Biogeografia do Bioma Cerrado: Vegetação e solos da Chapada dos Veadeiros. Brasília: Editora Universidade de Brasília, 256 p.

FLEMING TH. 1979. Do Tropical Frugivores Compete for Food? Am Zool 19: 1157-1172.

FrANKIE GW, BAKER HG AND OPLER PA. 1974. Comparative Phenological Studies of Trees in Tropical Wet and Dry Forests in the Lowlands of Costa Rica. J Ecol 62: 881-919.

Gentry H. 1993. A Field Guide to the Families and Genera of Woody Plants of Northwest South America (Colombia, Ecuador, Peru) with supplementary notes on herbaceous taxa. Chicago: University of Chicago Press, 920 p.

Heywood VH, Brummitt RK, Culham A and Seberg O. 2007. Flowering plant families of the world. Ontario: Firefly Books, 424 p.

KINOSHITA LS, TORRES RB, FORNI-MARTINS ER AND SPINELLI T. 2006. Composição florística e síndromes de polinização e de dispersão da mata do Sítio São Francisco, Campinas, SP, Brasil. Acta Bot Bras 20: 313-327.

KÖEPPEN W. 1948. Climatologia, con un Estudio de los Climas de la Tierra. México: Fondo de Cultura Economica, 478 p.

MARCo DE AND PAÉz SA. 2002. Phenology and phylogeny of animal-dispersed plants in a Dry Chaco forest (Argentina). J Arid Env 52: 1-16.

Marques MCM, Roper JJ AND SAlvalaggio APB. 2004. Phenological patterns among plant life-forms in a subtropical forest in southern Brazil. Plant Ecol 173: 203-213.
MARTINS GR. 2003. Arqueologia do Planalto Maracaju- Campo Grande. Brasília: Ministério da Integração Nacional, 255 p.

MENDONÇA RC, FELFILI JM, WALTER BMT, SILVA-JÚNIOR MC, REZENDE AV, FIlgueIRAS TS AND NOGUEIRA PEN. 1998. Flora vascular do Cerrado. In: SANO SM AND ALMEIDA SP (Eds), Cerrado: ambiente e flora, Planaltina: Embrapa-Cerrados, p. 287-556.

MIRANDA IS. 1995. Fenologia do estrato arbóreo de uma comunidade de Alter-do-Chão, PA. Rev Bras Bot 18: 235-240.

Monasterio M AND SARMiento G. 1976. Phenological strategies of plant species in the tropical savanna and the semi-deciduous forest of the Venezuelan Llanos. J Biogeogr 3: 325-356.

MOREIRA JLK. 2011. Anuário Interativo do Observatório Nacional. Available from: <http://euler.on.br/ephemeris/ index.php> Access in: 11 mar 2011.

Morellato LP AND Leitão-FILho HF. 1992. Padrões de frutificação e dispersão na Serra do Japi. In: MORELLATO LP (Ed), História Natural da Serra do Japi. Ecologia e preservação de uma área florestal no Sudeste do Brasil, Campinas: Editora da UNICAMP-FAPESP, p. 112-140.

Morellato LPC, ALBERTI LF AND HudSON IL. 2010. Applications of Circular Statistics in Plant Phenology: a Case Studies Approach. In: HUDSON IL AND KEATLEY MR (Eds), Phenological Research, Netherlands: Springer, p. 339-359.

MORELLATO LPC, LEITÃO-FILHO HF, RODRIGUES RRAND JOLY CA. 1990. Estratégias fenológicas de espécies arbóreas em floresta de altitude na Serra do Japi, Jundiaí, São Paulo. Rev Bras Biol 50: 149-162.

Morellato LPC, TAlora DC, TAKAhasi A, Bencke CC, Romera EC AND ZipParro VB. 2000. Phenology of Atlantic Rain Forest Trees: A comparative Study. Biotropica 32: 811-823.

Müller-Dombois D AND Ellemberg H. 1974. Aims and methods of vegetation ecology. New York: Wiley \& Sons, $547 \mathrm{p}$.

NASCIMENTO ART, Felfili JM AND MeIRELles EM. 2004 Florística e estrutura da comunidade arbórea de um remanescente de Floresta Estacional Decidual de encosta, Monte Alegre, GO, Brasil. Acta Bot Bras 18: 659-669.

OLIVEIRA JMZPS, PARANHOS-FILHO AC, CARRIJO MGG AND TORRES TG. 2006. Representação da Análise Ambiental de Área Ocupada por População Tradicional Quilombola (MS), Utilizando SIG e Sensoriamento Remoto. OLAM Cien \& Tecn 6: 219-252.

OlIVEIRA OS AND MARQUIS RJ. 2002. Introduction: Development of Research in the Cerrados. In: OLIVEIRA OS AND MARQUIS RJ (Eds), The Cerrados of Brazil. Ecology and Natural History of a Neotropical Savanna. New York: Columbia University Press, p. 1-12.

OLIVEIRA PE. 2008. Fenologia e biologia reprodutiva das espécies de Cerrado. In: SANO SM ET AL. (Eds), Cerrado: Ambiente e Flora. v.1. Planaltina: Embrapa-Cerrados, p. 273-287. 
Oliveira-Filho AT, Vilela EA, GaVILANES ML AND CARVALHO DA. 1994. Comparion of the Woody flora and soils of six áreas of montane semideciduous forest in Southern Minas Gerais, Brazil. Edinb J Bot 51: 355-389.

Opler PA, FrANKIE GW AND BAKER HG. 1976. Rainfall as a Factor in the Release, Timing, and Synchronization of Anthesis by Tropical Trees and Shrubs. J Biogeogr 3: 231-236.

VAN DER PIJL L. 1982. Principles of dispersal in higher plants. New York: Springer-Verlag, 214 p.

PInTO JRR AND OliveIRA-Filho AT. 1999. Perfil florístico e estrutura da comunidade arbórea de uma floresta de vale no Parque Nacional da Chapada dos Guimarães, Mato Grosso, Brasil. Rev Bras Bot 22: 53-67.

Pott A, Oliveira AKM, DAmasceno-Junior GA AND Silva JSV. 2011. Plant diversity of the Pantanal wetland. Braz J Biol 71(Suppl.): 265-273.

PRADO DE. 2000. Seasonally dry forests of tropical South America: from forgotten ecosystems to a New phytogeographic unit. Edinb J Bot 57: 437-461.

RADAMBRASIL. 1982. Projeto RADAMBRASIL. Levantamento de recursos naturais. Folha SF 21 Campo Grande: geologia, geomorfologia, pedologia, vegetação e uso potencial da terra. Ministério de Minas e Energia: Rio de Janeiro, 412 p.

RAMOS WM AND SARTORI ALB. 2013. Floristic Analysis and Dispersal Syndromes of Woody Species of the Serra de Maracaju, Mato Grosso do Sul, Brazil. Braz J Biol 73: 67-78.

RATTER AR, BRIDGEWATER S AND RIBEIRO JF. 2006. Biodiversity patterns of the Woody vegetation of the Brazilian Cerrado. In: PENNINGTON RT ET AL. (Eds), Neotropical Savannas and Seasonally Dry Forests: Plant Diversity, Biogeography and Conservation. Series 69. Florida: The Systematic Association Special Volume, p. 31-66.

RATTER JA, ASKEW GP, MONTGOMERY RF AND GIFFORD DR. 1978. Observations on forests of some mesotrophic soils in central Brazil. Rev Bras Bot 1: 47-58.

RATTER JA, BRIDGEWATER S AND RiBEIRo JF. 2003. Analysis of the floristic composition of the Brazilian Cerrado vegetation III: Comparison of the woody vegetation of 376 areas. Edinb J Bot 60: 57-109.

Reatto A, Correia JR, Spera ST and Martins ES. 2008. Solos do Bioma Cerrado. In: SANO SM ET AL. (Eds), Cerrado Ecologia e Flora, V.1. Planaltina: EmbrapaCerrados, p. 107-134.
RIBEIRO JF AND WALTER BMT. 2008. As principais fitofisionomias do Bioma Cerrado. In: SANO SM ET AL. (Eds), Cerrado Ecologia e Flora, V.1, Planaltina: EmbrapaCerrados, p. 151-199.

RoDRIGUES MT. 2005. A biodiversidade dos Cerrados: conhecimento atual e perspectivas, com uma hipótese sobre o papel das matas de galerias na troca faunística durante ciclos climáticos. In: SCARIOT A ET AL. (Eds), Cerrado: Ecologia, Biodiversidade e Conservação. Brasília: MMA, p. 235-246.

Ruggiero PGC, Batalha MA, Pivello VR and MeIRElles ST. 2002. Soil-vegetation relationships in Cerrado (Brazilian savanna) and semideciduous forest, Southeastern Brazil. Plant Ecol 160: 1-16.

Salis SM, Silva MP, Mattos PP, Silva JSV, PotT VJ AND PотT A. 2004. Fitossociologia de remanescentes de floresta estacional decidual em Corumbá, Estado do Mato Grosso do Sul, Brasil. Rev Bras Bot 27: 671-684.

SARMiento G AND Monasterio M. 1983. Life forms and phenology. In: BOURLIERE F (Eds), Ecosystems of the world - tropical savannas. Amsterdam: Elsevier, p.79-108.

Simon MF, GReTher R, QueIroz LP, SKemA C AND Pennington RT. 2009. Recent assembly of the Cerrado, a neotropical plant diversity hotspot, by in situ evolution of adaptations to fire. PNAS 106: 20359-20364.

TOWSEND CR, BEgON M AND HARPER JL. 2006. Fundamentos de ecologia. Segunda edição. Porto Alegre: Artmed, 592 p.

UNESCO. 1973. International classification and mapping of vegetation. Paris: UNESCO, 102 p.

VAsconcelos HL, VILHENA JMS, FACURE KG AND ALBERNAZ ALKM. 2010. Patterns of ant species diversity and turnover across $2000 \mathrm{~km}$ of Amazonian floodplain Forest J Biogeogr 37: 432-440.

WALTER H. 1986. Vegetação e zonas climáticas: tratado de ecologia global. In: WALTER H. (tradutoras: GIOVA AT AND BACKUP HT): Revisão técnica e notas Antonio Lamberti. São Paulo: EPU, 330 p.

WhitTAKER RH. 1975. Communities and ecosystems. $2^{\text {nd }}$ ed., New York: Collier-Macmillan Canada, Ltd.

ZAR JH. 1984. Bioestatical Analysis. $2^{\text {nd }}$ ed., New Jersey: Prentice Hall, 736 p. 